relationships between metabolic flux changes and the functional decline observed.

\section{P17 CYCLOSPORIN A MEDIATED INHIBITION OF THE MITOCHONDRIAL PERMEABILITY TRANSITION PORE (MPTP) ATTENUATES TIOTROPIUM BROMIDE MEDIATED CARDIOTOXICITY}

S Cassambai, S Dean, CJ Mee, A Hussain. Faculty Research Centre Applied Biological and Exercise Science (FRC-ABES), Coventry University, Coventry, UK

\subsection{6/heartjnl-2018-BSCR.22}

Muscarinic antagonists relieve bronchoconstriction due to the progressive condition of chronic obstructive pulmonary disease (COPD). Recent meta-analyses have highlighted increased stroke and myocardial infarction with the long acting muscarinic receptor antagonist, Tiotropium bromide. Opening of the mitochondrial permeability transition pore (mPTP) triggers cardiomyocyte death, therefore modulation of the pore could promote cardiomyocyte survival.

Isolated perfused rat hearts were subjected to ischaemia/ reperfusion $(\mathrm{I} / \mathrm{R})$ or normoxic protocols. Hearts were subjected to stabilisation, and perfusion \pm Tiotropium $(10 \mathrm{nM}-$ $0.1 \mathrm{nM})$, Cyclosporin A (CsA) $(200 \mathrm{nM})$ or Tiotropium $(1 \mathrm{nM}) \pm$ CsA. For $\mathrm{I} / \mathrm{R}$, regional ischaemia was induced following stabilisation. Hearts were stained using triphenyl-tetrazolium chloride (TTC) to determine infarct/risk ratios (\%). Data was analysed using one-way ANOVA and LSD, presented as mean \pm SEM.

All concentrations of Tiotropium significantly increased infarct/ risk ratio compared with controls. CsA decreased infarct/risk with respect to controls (Normoxia: $5.1 \pm 1.0 \%$ vs $10.3 \pm 1.9 \%$, $\mathrm{p}<0.05 ; \mathrm{I} / \mathrm{R}: \quad 7.2 \pm 1.2 \%$ vs $50.9 \pm 3.9 \%$ and $10.3 \% \pm 1.9 \%$, $\mathrm{p}<0.0001$ ), co-administration maintained this, with respect to Tiotropium $(1 \mathrm{nM})$ in normoxia, and also with control in $\mathrm{I} / \mathrm{R}$ (Normoxia: $8.4 \pm 2.1 \%$ vs $18.7 \pm 1.8 \%, \mathrm{p}<0.0001 ; \mathrm{I} / \mathrm{R}: 16.3$ $\pm 0.8 \%$ vs $65.4 \pm 3.0 \%$ and $50.9 \% \pm 3.9 \%, \mathrm{p}<0.0001)$.

This is the first pre-clinical study to suggest that Tiotropium increases infarct/risk ratio in an isolated perfused heart model via mPTP opening, as CsA decreases Tiotropiumand ischaemia/reperfusion-mediated myocardial injury. These findings suggest for a role of the mitochondria in mediating the adverse cardiac side-effects seen clinically.

\section{P18 ROLE OF CAMP in the regulation of Parkin-dependent mitophagy}

Miguel J Lobo, Manuela Zaccolo. Department of Physiology, Anatomy and Genetics, University of Oxford

\subsection{6/heartinl-2018-BSCR.23}

Parkinson's disease (PD) is characterised by compromised mitophagy, a highly specialised quality control process that removes dysfunctional mitochondria through a macroautophagy pathway. The proteins Parkin and PINK1 are key players in the mitophagic process. In healthy mitochondria with normal membrane potential, Parkin is located mainly in the cytosol, where its ubiquitin ligase activity is inhibited while PINK1 is imported into the mitochondria and becomes degraded by proteolysis. Following cellular stress, the depolarization of the mitochondrial membrane potential allows the stabilisation of PINK1 at the outer mitochondrial membrane (OMM), where it phosphorylates ubiquitin. This induces activation of Parkin and its translocation to damaged mitochondria, followed by mitophagy. Recent advances revealed that Parkin recruitment to depolarized mitochondria is severely inhibited by treatment with cAMP raising agents. cAMP-dependent activation of PKA has been shown to reduce PINK1 protein levels at the OMM through phosphorylation of MICOS (mitochondrial contact site and cristae organising system). Here we show that phosphodiesterase 2A2 (PDE2A2), a cAMP-degrading enzyme, interacts with components of the MICOS complex and regulates cAMP levels selectively at the mitochondria. Furthermore, our preliminary data show that in mouse embryonic fibroblasts deleted of PDE2A2 (MEF)PDE2A-/- the amount of Parkin recruited to the mitochondria is reduced compared to MEFWT under basal conditions. In agreement with these results, treatment with BAY 60-7550, a selective PDE2A inhibitor, promotes PKA-dependent phosphorylation of Mitofilin. In conclusion, we propose that PDE2A2 regulates a local cAMP pool at the mitochondria that leads to PKA-dependent phosphorylation of MICOS and Parkin recruitment to damaged mitochondria.

\section{P19 LOW LEVELS OF THE A3243G MTDNA MUTATION IN HUMAN INDUCED PLURIPOTENT STEM CELL- CARDIOMYOCYTES DO NOT CAUSE FUNCTIONAL OR METABOLIC DISTURBANCES BUT INCREASE WITH FURTHER PASSAGING}

${ }^{1} \mathrm{CP}$ Ricardo, ${ }^{1} \mathrm{~N}$ Hellen, ${ }^{1} \mathrm{G}$ Foldes, ${ }^{1} \mathrm{~T}$ Kodagoda, ${ }^{1} \mathrm{CM}$ Terracciano, ${ }^{2} \mathrm{MR}$ Duchen, ${ }^{1} \mathrm{SE}$ Harding. ${ }^{1}$ National Heart and Lung Institute, Imperial College, London, UK; ${ }^{2}$ Department of Cell and Developmental Biology, University College London, Gower Street, London, UK

\subsection{6/heartjnl-2018-BSCR.24}

The heteroplasmic mtDNA mutation A3243G can cause the mitochondrial condition MELAS. Mitochondrial replacement therapy can prevent transmission of mtDNA mutations to offspring but to maintain nuclear integrity, a certain amount of cytoplasm and mutated mtDNA is carried over $(<3 \%)$. It is unknown whether this will increase with age and this is particularly relevant in the heart, where mutations accumulate over time. We applied small molecule modulation of the Wnt/ $\beta$-catenin signalling pathway to generate pure populations of cardiomyocytes (CMs) from human induced pluripotent stem cells (hiPSCs) from a patient with $20 \%$ heteroplasmy for the A3243G mtDNA mutation No changes in the basal beating rate or time to peak and time to $50 \%$ relaxation were found. No differences in the response to $\beta$-adrenergic stimulation by isoprenaline or muscarinic inhibition by carbachol. A3243G hiPSC-CMs showed reduced excitability $(18.85 \pm 3.045 \mathrm{~ms}$ for control and $38.08 \pm 6.126 \mathrm{~ms}$ for A3243G, Mean \pm SEM, $\mathrm{p}=0.0084)$ but there were no changes in other calcium handling properties. Mitochondrial DNA copy number and both mitochondrial respiration and basal glycolysis were unaffected. We have seen a gradual increase in A3243G hiPSCs and derived CMs heteroplasmy with passaging (26.4\% to $38.7 \%$ over 6 passages). We conclude that A3243G heteroplasmy $<40 \%$ is not sufficient to affect the generation of hiPSC-CMs and their beating, calcium handling and metabolic properties. Having observed an increase in heteroplasmy with 